\title{
Fatigue and damage behaviour of CFRP elements in presence of wrinkles
}

\author{
by F. Panella*, A. Pirinu* and A. Saponaro* \\ * University of Salento, Department of Innovation Engineering, Lecce, Italy, authora@unisalento.it
}

\begin{abstract}
In this work, defect type "wrinkle" was characterized in CFRP notched specimens, performing fatigue and static experimental tests, coupled with non-destructive thermographic, optical and ultrasonic controls. This study includes an analysis of the damage to rupture behaviour of the material through the mechanical tensile test and a non-destructive inspection procedure is adopted during HCF load, in order to detect in real time local compliance variations and damage initiation. Thermographic continuous monitoring and occasional ultrasonic analysis are implemented with the purpose to analyse composite anomalies during fatigue life and elaborate a method for the identification of the wrinkle induced failure.
\end{abstract}

\section{Introduction}

Structural health of mechanical structures depends on manufacturing process, non-destructive detection and material defect characterization represent the main useful procedure for prediction of mechanical decay caused by local composite interlaminar damage.

Numerous papers are found on composite defects types, from which fatigue damage originates, such as porosities and small delaminations, however recent studies examined mechanical behaviour of wrinkle samples, defined as orientation variation of composite laminate in various dimension scale [1]. This type of defect arises during processes in which the composite is subjected to an incorrect layout of layers or thermal gradients and / or differential in pressures applied to the layers of the composite [2,3].

Since wrinkle defect is a defect type hard to detect and characterize [4], is important to monitor some indirect parameters (displacement field, temperature variation or ultrasonic signal amplitude perturbations during load [5]; therefore amplitude of fundamental frequency, stiffness variation and fatigue life reduction were studied on different samples to evaluate damage evolution induced by fatigue tests and to give general indications on suitable ND approaches and data analysis to follow material degradation for CFRP samples in presence of wrinkles [6].

In this work, experimental controls methodology for delamination evolution analysis on unidirectional CFRP elements under fatigue is proposed, since Fibre-reinforced composite structures under fatigue loads exhibit continuing damage accumulation and degradation of effective mechanical properties are generally observed, but the presence of wrinkles around notched zone of laminate is to be assessed. Since advanced composites application are to be applied after extensive experimental certification tests, the proposed work is based on combined experimental and digital image correlation (DIC) under static load of the same specimen, to try correlation of superficial strain with compliance variation and eventually ND control data[6].

Experimental results indicated fatigue life of CFRP elements obviously decreased with the increase of stress levels, but the same cannot be said about damage evolution levels and structural specimen state before final rupture, as well as the amount of residual static strength and stiffness after fatigue testing on specimen apparently still intact.

Experimental thermographic and ultrasonic results are elaborated with MATLAB analysis for damage analysis of various specimens under fatigue and results are implemented to check results with strain and compliance data acquired during tests. Authors make use of open source platform Ncorr-2D-DIC in order to obtain displacement and strains on specimen surface under study; data elaboration and post-processing of all experimental results are discussed. 

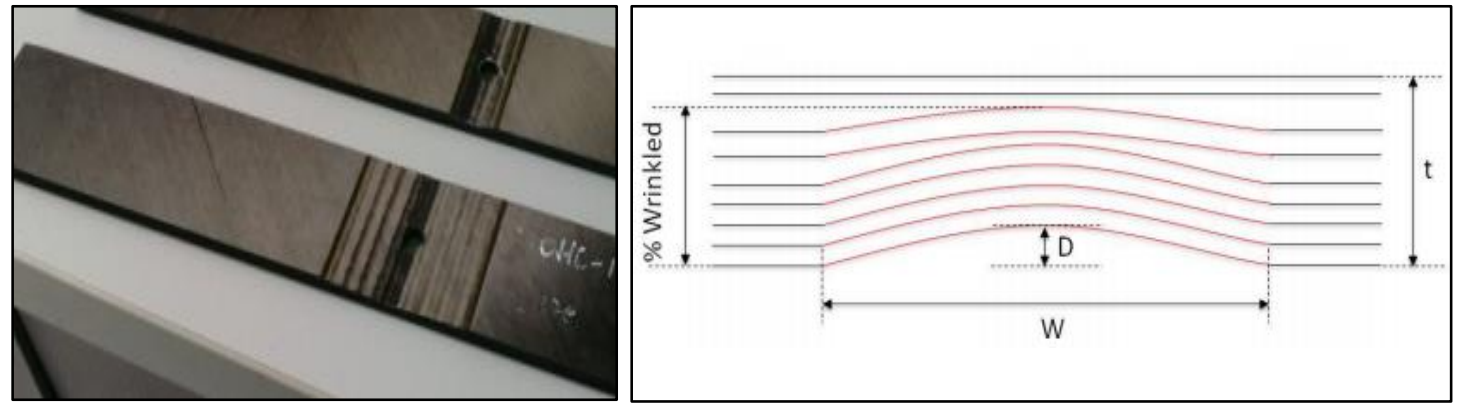

Fig. 1. Example wrinkle samples and wrinkles geometry.
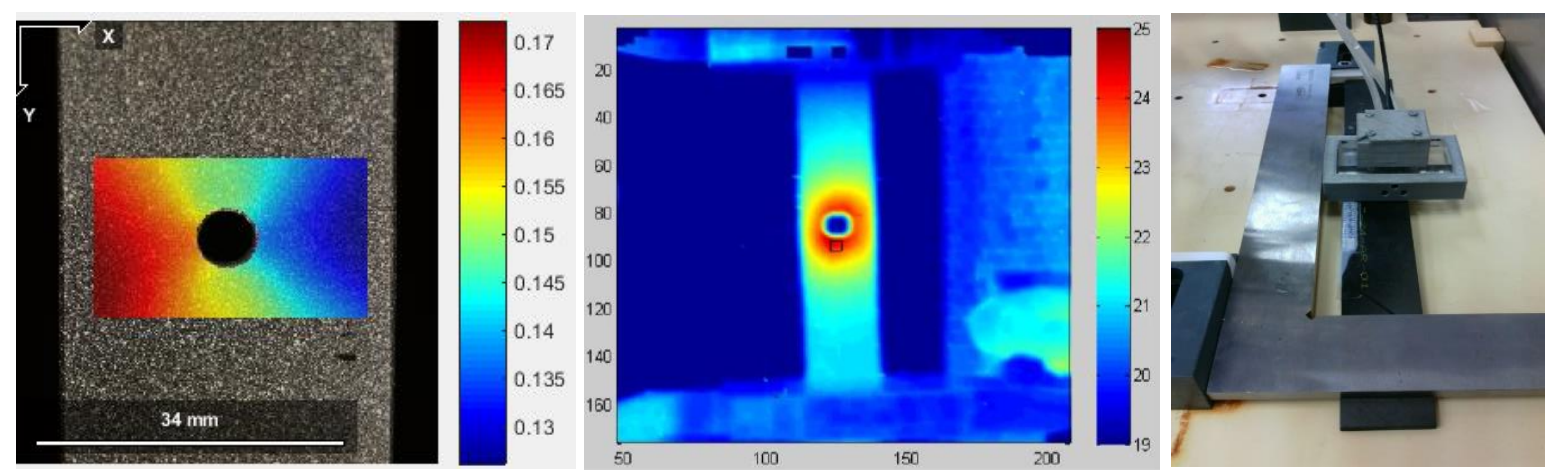

Fig. 2. DIC strain field, thermographic temperatures and ultrasonic procedures on CFRP samples.

\section{REFERENCES}

[1] Liang B., Hamila N., Peillon M., Boisse P., Analysis of thermoplastic prepreg bending stiffness during manufacturing and of its influence on wrinkling simulations; Composites Part A: Applied Science and Manufacturing; 67: pp.111-122, 2014.

[2] Lightfoot J. S., Wisnom M. R., Potter K., A new mechanism for the formation of ply wrinkles due to shear between plies; Composites Part A Applied Science and Manufacturing; 49: pp.139-147, 2013.

[3] Pandey R.K., Sun C.T, Mechanisms of wrinkle formation during the processing of composite laminates; Composites Science and Technology; 59: pp. 405-417, 1999.

[4] Christian Garnier, Marie-Laetitia Pastor, Florent Eyma, Bernard Lorrain; The detection of aeronautical defects in situ on composite structures using Non-Destructive Testing; Composite Structures; 2011.

[5] Fernández-López A., Larrañaga-Valsero B., Guemes A.; Wrinkle detection with Ultrasonic Phased Array technology; Department of Aeronautics, Polytechnic University of Madrid (UPM), Spain; 2014.

[6] Elhajjar R. F., Shams S. S., Gabor J. Kemeny, Stuessy G.; A hybrid numerical and imaging approach for characterizing defects in composite structures; Composites Part A: Applied Science and Manufacturing; 2016. 